\title{
1 Functional analysis of the three major PGRPLC isoforms in the 2 midgut of the malaria mosquito Anopheles coluzzii
}

\author{
3 Faye H. Rodgers ${ }^{1, a}$, Julia A. Cai ${ }^{1}$, Andre N. Pitaluga ${ }^{1, b}$, Dominique Mengin- \\ 4 Lecreulx $^{2}$, Mathilde Gendrin ${ }^{1,3}$ and George K. Christophides ${ }^{1 *}$ \\ ${ }^{1}$ Department of Life Sciences, Imperial College London, Exhibition Road, London SW7 2AZ, UK. \\ ${ }^{2}$ Institute for Integrative Biology of the Cell (I2BC), CEA, CNRS, Univ Paris-Sud and Université Paris- \\ 8 Saclay, 91198, Gif-sur-Yvette, France \\ ${ }^{3}$ Institut Pasteur de la Guyane, BP6010 Cayenne, French Guiana, France \& Department of Parasites \\ and Insect Vectors, Institut Pasteur, Paris, France
}

*Corresponding author: g.christophides@imperial.ac.uk

\section{Footnotes}

14 a Current address: Wellcome Sanger Institute, Wellcome Genome Campus, Hinxton, Cambridge CB10 15 1SA, UK

${ }^{\text {b } C u r r e n t ~ a d d r e s s: ~ I n s t i t u t o ~ O s w a l d o ~ C r u z ~-~ F I O C R U Z, ~ A v . ~ B r a s i l ~ 4365, ~ R i o ~ d e ~ J a n e i r o, ~ B r a z i l ~}$

\begin{abstract}
Peptidoglycan recognition proteins (PGRPs) constitute the primary means of bacterial recognition in insects. Recent work in the model organism Drosophila has revealed the mechanisms by which the complement of PGRPs refine the sensitivity of different tissues to bacterial elicitors, permitting the persistence of commensal bacteria in the gut whilst maintaining vigilance against bacterial infection. Here, we use in vivo knockdowns and in vitro pull-down assays to investigate the role of the three major isoforms of the transmembrane receptor of the Imd pathway, PGRPLC, in basal immunity in the Anopheles coluzzii mosquito midgut. Our results indicate that the mosquito midgut is regionalized in its expression of immune effectors and of PGRPLC1. We show that PGRPLC1 and PGRPLC3 are pulled down with polymeric DAP-type peptidoglycan, while PGRPLC2 and PGRPLC3 co-precipitate in the presence of TCT, a peptidoglycan monomer. These data suggest that, as found in Drosophila, discrimination of polymeric and monomeric PGN by Anopheles PGRPLC participates in the regulation of the Imd pathway.
\end{abstract}

\section{Introduction}

In insects, bacteria are mainly perceived by the detection of the bacterial cell wall component peptidoglycan (Leulier et al. 2003). Upon recognition, the Imd pathway is one of the major means of signal transduction to initiate antibacterial responses. Drosophila Imd pathway activation is tightly regulated in a tissue specific manner, with the gut epithelium being adapted to tolerate the presence of the microbiota. This is achieved through several mechanisms including regulation of ligand availability (Paredes et al. 2011; Zaidman-Remy et al. 2006; Costechareyre et al. 2016), regulation of receptor signaling capacity (Basbous et al. 2011; Maillet et al. 2008; Aggarwal et al. 2008; Kleino et al. 2008; Neyen et al. 2016) and regulation of effector gene transcription (Ryu et al. 2008). 
Drosophila also employs different peptidoglycan recognition proteins (PGRPs) in different tissues as immune receptors or regulators. A diversity of PGRP receptors allows discrimination of the type, the polymerisation status and the level of peptidoglycan that is able to stimulate antimicrobial gene expression (Bosco-Drayon et al. 2012; Neyen et al. 2012). Transmembrane PGRP-LC (FBgn0035976) encodes six isoforms resulting from a combination of 3 splice variants in the extracellular domains (PGRP-LCX, PGRP-LCa and PGRP-LCy) and 2 splice variants in the intracellular domain (Neyen et al. 2016). PGRP-LCx binds both polymeric and monomeric peptidoglycan whilst PGRP-LCa has no peptidoglycan binding capacity (Chang et al. 2005; Mellroth et al. 2005). The pathway can be stimulated by either polymeric peptidoglycan inducing PGRP-LCx-LCx homodimerization or by TCT (tracheal cytotoxin, a peptidoglycan monomer released by growing bacteria) inducing PGRP-LCx-LCa heterodimerization (Chang et al. 2005; Chang et al. 2006; Kaneko et al. 2004; Neyen et al. 2012). PGRP-LCx is shown to be necessary and sufficient for resistance to systemic infection by Gramnegative bacteria, indicating that polymeric peptidoglycan is the primary immune elicitor in the haemolymph, with PGRP-LCa-dependent TCT-induced dimers amplifying the strength of the response (Neyen et al. 2012). The function of PGRP-LCy is less well established (Kaneko et al. 2004; Werner et al. 2003), but could be related to recognition of other types of pathogen-associated molecular patterns (PAMPs) such as the microbial metabolite acetate (Kamareddine et al. 2018). The intracellular splicing variant including exon 4 encodes Imd signaling-inducing PGRP-LC, while the variant including exon 5 encodes regulatory PGRP-LC (rPGRP-LC), which sends PGRP-LC to degradation upon specific sensing of polymeric peptidoglycan (Neyen et al. 2016). This downregulation of the Imd pathway via receptor degradation is considered as a way to resolve Imd activation while polymeric peptidoglycan released by lysed bacteria is still present in the haemolymph.

A second Imd pathway receptor, PGRP-LE (FBgn0030695), is localized intracellularly where it binds TCT, inducing receptor oligomerization and Imd pathway activation (Kaneko et al. 2006; Lim et al. 2006). PGRP-LE is strongly enriched in the Drosophila midgut (excluding the cardia) where it is thought to play a significant role in detecting live bacteria and TCT (Bosco-Drayon et al. 2012; Neyen et al. 2012). This PGRP compartmentalization reflects a fine-tuning of the responsiveness of different tissues to their different levels of bacterial exposure. The haemolymph, being a nominally sterile environment, uses extracellular receptors to be highly responsive to both polymeric peptidoglycan and TCT. The posterior midgut, meanwhile, relies largely on an intracellular TCT receptor; here, the Imd pathway is stimulated only when bacterial growth reaches such a threshold that TCT is transported intracellularly. This is appropriate for a tissue that is in constant contact with microbiota.

Anopheles mosquitoes also rely on the Imd pathway for their response to bacterial infection and for controlling microbiota load, which increases significantly following blood feeding (Meister et al. 2009; Dong, Manfredini, and Dimopoulos 2009). A. gambiae PGRPLC (AGAP005203) has a similar genetic architecture to its Drosophila orthologue, encoding three main isoforms (PGRPLC1, PGRPLC2 and PGRPLC3) that vary in their extracellular PGRP domains. These PGRP domains have derived from independent duplications between the mosquito and fruit fly lineages, which have been therefore assumed to acquire different functions (Christophides et al. 2002). It has been reported that all mosquito isoforms contribute to resistance to systemic Gram-negative infection, whilst PGRPLC1 and PGRPLC3 only are necessary for resistance to Gram-positive infection (Meister et al. 2009). Additionally, in two mosquito derived cell lines it is found that PGRPLC1 is the only isoform that is necessary for induction of the antimicrobial peptide (AMP) CEC1 following bacterial challenge (Lin et al. 2007). Overexpression of PGRPLC1 is found to be sufficient for CEC1 induction even in the absence of bacterial challenge, with PGRPLC3 overexpression having a similar but milder effect (Lin et al. 2007). A non-peptidoglycan-binding PGRP, PGRPLA, is also known to be a positive regulator of the pathway in the gut (Gendrin et al. 2017). Notably, A. gambiae does not have a PGRP-LE orthologue. A model has not so far emerged regarding the functions of the different PGRPLC ectodomains in the $A$. gambiae immune response. gambiae genome is lacking in several of the characterized regulators in Drosophila, we investigated 
the role of PGRPLC in basal immune induction in the mosquito gut. We show that the midgut of $A$. coluzzii (called $A$. gambiae M molecular form in earlier studies of PGRPLC) is compartmentalized in its expression of the immune effector-encoding transcripts GAM1, CEC1 and LYSC1, and of one of the PGRPLC isoforms, PGRPLC1. Using isoform specific knockdown, we show that PGRPLC2 and PGRPLC3 both positively regulate REL2-responsive AMPs in the midgut. Functional analyses of recombinant PGRPLC domains indicate that PGRPLC1 and PGRPLC3 are pulled down with polymeric DAP-type peptidoglycan, whilst in the presence of TCT PGRPLC2 and PGRPLC3 coprecipitate.

\section{Materials and Methods}

99 Mosquito rearing, blood feeding and antibiotic treatment

The $A$. coluzzii N'gousso colony was maintained at $27{ }^{\circ} \mathrm{C}\left( \pm 1^{\circ} \mathrm{C}\right), 70-80 \%$ humidity with a $12 \mathrm{~h}$ light/dark cycle. All adults were allowed ad libitum access to $5 \% \mathrm{w} / \mathrm{v}$ fructose solution and females were maintained on human blood. 2-3 day old female mosquitoes were used in all experiments, having been exposed to only fructose solution ('sugar-fed'), or $24 \mathrm{~h}$ after taking a human blood meal ('blood-fed'). In antibiotic treatments, the blood meal and sugar solutions were supplemented with 60 $\mathrm{U} / \mathrm{ml}$ penicillin, $60 \mu \mathrm{g} / \mathrm{ml}$ streptomycin and $50 \mu \mathrm{g} / \mathrm{ml}$ gentamicin. Antibiotic treatment efficacy was confirmed by qRT-PCR against $16 \mathrm{~S}$ rRNA.

\section{Gene expression and microbiota analysis}

qRT-PCR was used to assess expression levels of AMPs (CEC1, GAM1 and LYSC1), PGRPLC transcripts and $16 \mathrm{~S}$ rRNA load. Midgut tissues were dissected into subregions using a thin needle. For posterior tissue sections, either the whole posterior region (referred to as "posterior") was sampled, or each half of the posterior region (referred to as "proximal posterior" and "distal posterior") was sampled separately, as indicated. Tissues were flash frozen on dry ice and homogenized in TRIzol (Invitrogen) using a Precellys 24 homogenizer (Bertin) with 0.5-mm-wide glass beads (Bertin) or with a pestle-based motorized homogenizer. RNA was extracted according to the TRIzol (Invitrogen) manufacturer's instructions and resuspended in molecular-biology grade, RNase-free water. cDNA was synthesized using PrimeScript Reverse Transcriptase (Takara) or SuperScript III Reverse Transcriptase (ThermoFisher Scientific). qRT-PCR was performed on a 7500 Fast Real Time PCR machine (Applied Biosystems) using a SYBR premix ex Taq kit (Takara). The ribosomal transcript S7 (AGAP010592) was used as normalization control. qRT-PCR primer sequences are listed in Table S1, and PGRPLC isoform-specific amplicons are represented Fig S1A.

\section{Double stranded RNA preparation and gene knockdown}

122 Double stranded RNA (dsRNA) was used for transient in vivo knockdown of target genes by RNAi. 123 The target region was amplified from total cDNA using primers flanked with the T7 RNA polymerase promoter sequence (primer sequences listed in Table S1) with Phusion Taq polymerase (NEB). PCR products were purified using the QIAquick PCR purification kit (Qiagen). dsRNA was then synthesized from the PCR product by overnight incubation at $37{ }^{\circ} \mathrm{C}$ with $\mathrm{T} 7$ polymerase and dNTPs from the MEGAscript RNAi kit (ThermoFisher Scientific). dsRNA was purified using the RNeasy kit (Qiagen) and adjusted to a concentration of $6000 \mathrm{ng} / \mu \mathrm{l}$. $69 \mathrm{nl}$ of $6000 \mathrm{ng} / \mu \mathrm{l}$ dsRNA (totalling $414 \mu \mathrm{g}$ ) was injected into the thorax of $\mathrm{CO}_{2}$-anaesthetised 0-2-day old female mosquitoes using the Nanoject II (Drummond Scientific). dsRNA against a region of the bacterial lac operon (dsLACZ) was injected as a control. 
133 PGRP ectodomains were amplified from an A. coluzzii cDNA library by PCR with Phusion polymerase 134 (NEB). The amplified products of the appropriate size were gel extracted using the QIAquick gel 135 extraction kit (Qiagen), cloned by ligation independent cloning using the Ek/LIC cloning kit (Novagen) 136 into the plEX-10 vector (Novagen) for insect cell expression and verified by sequencing. pIEX-10 137 introduces an N-terminal strep tag II, a C-terminal His-tag and a secretion signal peptide.

\section{Transfection and stable cell line generation}

Sf9 lepidopteran cells were maintained at $27^{\circ} \mathrm{C}$ in adherent culture in SF900 II serum free medium. Three stable cell lines were generated, expressing PGRPLC1, PGRPLC2 and PGRPLC3 ectodomains respectively. For transfection, cells were seeded at $80 \%$ confluency in $2.5 \mathrm{ml}$ SF900 II medium. $2 \mu \mathrm{g}$ pIEX-10+insert and $0.2 \mu \mathrm{g}$ PIE1- neo (Novagen) were added dropwise with $8 \mu \mathrm{g}$ Cellfectin II reagent (Invitrogen). PIE1-neo expresses the neo gene, facilitating selection in the presence of the antibiotic G418. $24 \mathrm{~h}$ post transfection, the medium was replaced with complete medium (SF900 II plus $10 \%$ fetal calf serum (FCS)) and cells were re-plated in serial dilution. After $36 \mathrm{~h}$, the medium was changed to complete medium plus $1 \mathrm{mg} / \mathrm{ml} \mathrm{G} 418$. This selective medium was refreshed every 4 days for approximately 2 weeks, or until resistant colonies were observed. Selection was reduced to $0.3 \mathrm{mg} / \mathrm{ml}$ G418 in complete medium and this concentration was maintained throughout. Once the selected cells reached confluency, a sample of conditioned medium was analyzed by Western blot for expression of recombinant protein.

\section{Recombinant protein purification}

152 Recombinant proteins were purified from conditioned medium by His-tag affinity. TALON beads 153 (Takara; approx. $250 \mu \mathrm{l}$ per $150 \mathrm{ml}$ conditioned medium) were equilibrated with two washes in 10 154 volumes of equilibration buffer $\left(50 \mathrm{mM} \mathrm{NaH}_{2} \mathrm{PO}_{4}, 300 \mathrm{mM} \mathrm{NaCl}, \mathrm{pH} 7.0\right)$. The beads were incubated 155 with conditioned medium, rotating at room temperature, for $2 \mathrm{~h}$. Beads were then washed twice with 20 volumes of wash buffer $\left(50 \mathrm{mM} \mathrm{NaH}_{2} \mathrm{PO}_{4}, 300 \mathrm{mM} \mathrm{NaCl}, 10 \mathrm{mM}\right.$ imidazole, $\mathrm{pH}$ 7.0) for $15 \mathrm{~min}$ followed by one wash with 5 volumes of wash buffer for $5 \mathrm{~min}$, both at room temperature. His-tagged proteins were eluted in elution buffer $\left(50 \mathrm{mM} \mathrm{NaH}_{2} \mathrm{PO}_{4}, 300 \mathrm{mM} \mathrm{NaCl}, 150 \mathrm{mM}\right.$ imidazole, $\mathrm{pH}$ 7.0). Purified proteins were then dialyzed (Amicon Ultra $4 \mathrm{ml}$ dialyzer, $10 \mathrm{kDa}$ pore size) overnight at $4{ }^{\circ} \mathrm{C}$ into wash buffer to remove the imidazole for further processing. Where specified, the C-terminal Histag was cleaved with ProTEV plus protease (Promega), using the TEV cleavage site that was introduced with the reverse primers during cloning. Approximately $200 \mu \mathrm{g}$ recombinant protein was incubated with ProTEV cleavage buffer, $1 \mathrm{mM}$ DTT and 100 units of ProTEV protease for $3 \mathrm{~h}$ at $30^{\circ} \mathrm{C}$. Cleaved protein was isolated from the His tag and the protease itself, which also has a His-tag, using TALON beads as described above (retaining the flow through).

\section{Peptide:N-glycosidase (PNGase F) treatment}

$5 \mu \mathrm{g}$ recombinant protein was incubated in denaturation buffer $(0.02 \%$ SDS, $10 \mathrm{mM} \beta-$ mercaptoethanol) at $100{ }^{\circ} \mathrm{C}$ for 10 min before being incubated with 2.5 units PNGase $\mathrm{F}$ and $0.8 \%$ Triton X-100 at $37^{\circ} \mathrm{C}$ for $3 \mathrm{~h}$.

\section{Western blotting}

171 After separation by SDS-PAGE, proteins were transferred to a nitrocellulose membrane by semi dry 172 transfer (15 V for $30 \mathrm{~min}$ ) and membranes blocked in phosphate buffered saline plus $1 \%$ Tween-20 173 (PBST) with $3 \%$ bovine serum albumin (BSA), either overnight at $4{ }^{\circ} \mathrm{C}$ or for $1 \mathrm{~h}$ at room temperature. Primary antibody was added in fresh blocking buffer at the specified concentration and incubated overnight at $4{ }^{\circ} \mathrm{C}$. After three $10 \mathrm{~min}$ washes in PBST, blots were incubated with the specified secondary antibody conjugated with horseradish peroxidase (HRP) at a 1:10000 dilution in PBST with 
$3 \%$ BSA for $1 \mathrm{~h}$ at room temperature, before three further washes in PBST. Blots were exposed with

178 ECL chemiluminescence substrate (Pierce) and visualized with a Biorad Chemidoc Imager.

\section{Peptidoglycan preparation}

180 DAP-type peptidoglycan polymer was purified from the E. coli BW25113 $\Delta / p p:: \mathrm{Cm}^{\mathrm{R}}$ strain that does 181 not express the Lpp lipoprotein, as described (Leulier et al. 2003). The GlcNAc-MurNAc(anhydro)-L182 Ala-D-iGlu-meso-DAP-D-Ala peptidoglycan monomer fragment (designated as TCT, tracheal 183 cytotoxin) was obtained by peptidoglycan digestion with SItY transglycosylase and purified by HPLC 184 as previously described (Stenbak et al. 2004).

185

186

187

188

189

190

191

\section{2}

193

194

195

196

197

198

\section{Peptidoglycan co-precipitation assay}

Recombinant protein $(100 \mu \mathrm{g} / \mathrm{ml})$ was incubated with insoluble peptidoglycan from Escherichia coli (1 $\mathrm{mg} / \mathrm{ml})$ in $50 \mu \mathrm{l}$ binding buffer $(150 \mathrm{mM} \mathrm{NaCl}, 20 \mathrm{mM}$ HEPES, $\mathrm{pH} 7.5)$ for $1 \mathrm{~h}$ at room temperature, rotating. The insoluble peptidoglycan was then pelleted by centrifugation (13000 g, $5 \mathrm{~min})$. The pellet was washed three times in $1 \mathrm{ml}$ binding buffer and resuspended in $50 \mu \mathrm{l}$ binding buffer. Pellet and supernatant fractions were analyzed by Western blot, using a primary antibody against the $\mathrm{N}$-terminal strep tag II.

\section{PGRPLC co-precipitation assay}

For protein-protein co-precipitation assays, recombinant protein after cleavage of the His-tag (100 $\mu \mathrm{g} / \mathrm{ml})$ was incubated with recombinant protein that retained its His-tag $(100 \mu \mathrm{g} / \mathrm{ml})$ in the presence of an approximately 5-fold molar excess of TCT $(20 \mu \mathrm{M})$ in $50 \mu \mathrm{l}$ binding buffer for $1 \mathrm{~h}$ at room temperature, rotating. His-tagged protein and any interacting protein were then purified by His-tag affinity. The flow through and bead bound fractions were analyzed for their protein content by Western blot, probing for the $\mathrm{N}$-terminal strep tag II.

\section{Statistical analysis}

qRT-PCR expression data (including $16 \mathrm{~S}$ analysis) were analyzed with linear mixed effect regression models using the Ime4 package in $\mathrm{R}$ (version 3.1.2). Mosquito batch (i.e., experimental replicate) was included as a random effect, with tissue section and/or dsRNA as fixed effects. When examining the effect of dsRNA in different tissues, a tissue:dsRNA interaction term was fitted first (together with the main effect terms). If this significantly improved the fit of the model (as assessed by ANOVA), we go on to report the effect of dsRNA in each tissue independently (expression dsRNA + (1/replicate)). Otherwise, the reported $p$ value is the result of an ANOVA test after removing dsRNA from the full model (expression $\sim$ tissue + dsRNA + (1|replicate)).

\section{Results}

\section{AMP expression and PGRPLC1 expression are spatially heterogeneous along the mosquito midgut}

We examined whether the $A$. coluzzii gut tissue is spatially heterogeneous in its immune activity, and how this putative heterogeneity might be related to the presence of the gut microbiota. Midguts were micro-dissected into three main regions: the cardia, anterior and posterior (Fig 1A). The abundance of bacterial 16S rRNA and the expression of transcripts of the microbiota-dependent antimicrobial peptides (AMPs; Rodgers et al. 2017) CEC1 (AGAP000693), GAM1 (AGAP008645) and LYSC1 (AGAP007347) were analyzed by qRT-PCR. We observed that bacterial proliferation upon blood feeding is limited to the posterior section of the gut, the region which harbours the blood bolus (Fig 1B). Strikingly, all three AMPs analyzed were heterogeneously expressed across the length of both 
sugar-fed and blood-fed guts, with expression being higher in the cardia and anterior regions than the posterior (Fig 1C-E), despite the abundance of the microbiota in this region after blood feeding. We assessed whether all regions of the gut are able to respond transcriptionally to the microbiota by comparing AMP expression in control mosquitoes with mosquitoes that have been treated with a robust cocktail of antibiotics (Fig 1C-E). Expression of one AMP (GAM1 in the sugar-fed posterior region) or all three AMPs (in all other regions) was lower after antibiotic treatment; thus, we conclude that the whole length of the gut epithelium is able to respond to microbiota-derived bacterial elicitors.

We next analyzed the expression pattern of the three major PGRPLC isoforms. We found that the expression of PGRPLC1 correlates well with that of the AMPs analyzed, being more highly expressed in the cardia and anterior regions than in the posterior (Fig 1F). PGRPLC2 and PGRPLC3 do not vary in their expression across the length of the gut (Fig S1). Altogether, these data show that the expression of CEC1, GAM1, LYSC1 and PGRPLC1 vary significantly along the length of the gut, though their expression pattern appears to be uncoupled from levels of exposure to commensal bacteria in the different regions.

\section{PGRPLC2 and PGRPLC3 are positive regulators of REL2-responsive AMPs in the midgut}

Given that PGRPLC-dependent activation of the Imd pathway plays a role in controlling the mosquito gut microbiota (Dong, Manfredini, and Dimopoulos 2009; Meister et al. 2009), we next sought to decipher which PGRPLC isoforms are responsible for stimulating Imd activity in the midgut.

We first determined which AMPs are transcriptionally responsive to the Imd pathway in the sugar-fed and blood-fed gut by monitoring the expression of GAM1, LYSC1 and CEC1 after injection of dsRNA against the Imd pathway transcription factor REL2 (AGAP006747) (Fig 2, Fig S2, Fig S3). Of the AMPs analyzed, we found GAM1 expression to be responsive to REL2 knockdown across the sugarfed gut ( $p<0.05$, Fig 2A). REL2 knockdown had no significant effect on CEC1 or LYSC1 expression in the sugar-fed gut (Fig S3A-B).

We next injected dsRNA against each specific PGRPLC ectodomain, trying to recapitulate the effect on GAM1 expression (Fig 2A). As observed previously (Meister et al. 2009), we achieved limited and variable knockdown efficiency against individual PGRPLC isoforms (Fig S2). Nevertheless, we found that PGRPLC2 dsRNA caused a significant reduction in GAM1 expression in the cardia $(\mathrm{p}<0.05)$ and anterior $(p<0.05)$ regions of the sugar-fed midgut. PGRPLC3 dsRNA had no effect in any region of the sugar-fed midgut. PGRPLC1 dsRNA resulted in a significant increase in GAM1 expression in the anterior region $(p<0.05)$, but interestingly not in the cardia despite this region having very high PGRPLC1 expression.

After blood feeding, GAM1 remained responsive to REL2 throughout the gut ( $p<0.05$, Fig $S 3 C$ ), though no PGRPLC isoforms had any effect on GAM1 expression. We also observed a significant reduction in LYSC1 expression upon REL2 knockdown in the cardia $(p=0.05)$ and anterior $(p<0.05)$ regions, with the posterior region showing the same trend (Fig 2B). Concurrently, we found PGRPLC2 dsRNA to cause a reduction in $L Y S C 1$ expression in the posterior region $(p<0.05)$ and PGRPLC3 dsRNA to cause a reduction in $L Y S C 1$ expression in the cardia $(p<0.05)$ and posterior $(p<0.01)$ regions.

Altogether, these data confirm that the AMPs GAM1, CEC1 and LYSC1 are REL2-responsive in at least some regions of the midgut. PGRPLC2 and PGRPLC3 also both act as positive regulators of two of these AMPs. Despite PGRPLC1 being known to be able to induce the Imd pathway (Lin et al. 2007), we did not find evidence for this isoform acting as a positive Imd regulator in the midgut. Our data rather suggests that PGRPLC1 could be acting as a negative regulator, though we cannot rule out that PGRPLC1-dependent signaling to other pathways induces dysbiosis, causing the observed induction of GAM1 expression. 
In order to understand further the differing functions of the PGRPLC isoforms, their PGRP domains were recombinantly produced and subjected to in vitro functional analysis. The recombinant PGRP domains of the three major isoforms were secreted from Sf9 lepidopteran cells and purified from conditioned media with a C-terminal His-tag (Fig S4A). The His-tags were cleaved before further functional analysis to avoid artefactual interaction between them and peptidoglycan (Basbous et al. 2011; Persson, Oldenvi, and Steiner 2007). PGRPLC1 and PGRPLC3 were observed to migrate as a double and triple band, respectively, whilst PGRPLC2 migrates as a single band (Fig S4B). We hypothesized that this may be due to glycosylation, a common posttranslational modification in secreted and membrane bound proteins. All of the ectodomains have predicted O-glycosylation sites, whilst PGRPLC1 and PGRPLC3 additionally have two and three predicted N-glycosylation sites, respectively (Fig S4C). Treatment with PNGase $\mathrm{F}$, an N-glycosidase, indeed resulted in a shift in the migration of PGRPLC1 and PGRPLC3 with no effect on PGRPLC2 (Fig S4D), suggesting that PGRPLC1 and PGRPLC3 undergo N-glycosylation.

We first looked at the ability of the three PGRPLC ectodomains to interact with peptidoglycan. As the vast majority of bacteria present in the gut microbiota are Gram-negative, we used DAP-type peptidoglycan purified from E. coli for this purpose. Each PGRPLC isoform was incubated with insoluble peptidoglycan, and the supernatant and washed pellet analyzed by Western blot. Under the conditions used, PGRPLC1 and PGRPLC3 were both pulled down with the polymeric peptidoglycan, whilst PGRPLC2 remained in the supernatant fraction (Fig 3A). When isoforms were combined pairwise and co-precipitated with peptidoglycan, we observed a protein migrating as a single band (likely PGRPLC2) in the supernatant fraction of both the PGRPLC1/PGRPLC2 and PGRPLC2/PGRPLC3 combinations (Fig 3B). Whilst we cannot rule out some level of PGRPLC2peptidoglycan interaction, we conclude that PGRPLC1 and PGRPLC3 co-precipitate with polymeric DAP-type peptidoglycan, both alone and in the presence of other PGRPLC isoforms.

\section{PGRPLC2 and PGRPLC3 co-precipitate in the presence of TCT}

Next, we considered which of the PGRP domains could interact with one another in the presence of the peptidoglycan monomer, TCT. To do this, we combined one isoform with its His-tag with another isoform whose His-tag had been cleaved off, in the presence of TCT. Purifying by His-tag affinity, we then pulled down the His-tagged protein and observed whether the other, non-His tagged, isoform pulled down with it or remained in the flow-through fraction (Fig 4A). When probing initially with antistrep tag II, we found that the PGRPLC2/PGRPLC3 combination led to near complete pull down (Fig 4A), with no free PGRPLC3 being observed in the flow-through fraction. This was not due to residual His-tag being retained on PGRPLC3, as in combination with both PGRPLC3(His) and PGRPLC1(His) a large proportion of PGRPLC3 is found in the flow-through fraction. To validate this finding, we performed the reciprocal experiment, pulling down PGRPLC1 and PGRPLC2 with PGRPLC3(His) (Fig 4B). As expected, PGRPLC2 appears almost entirely in the bound fraction whilst PGRPLC1 appears mostly in the free fraction, again with a small proportion in the bound fraction. In addition to the PGRPLC2/LC3 interaction, we also observed the appearance of bands at sizes that correspond to the presence of the His-tag. We cannot therefore rule out these dimers being an artefact caused by the presence of the His-tag, a proposition that has some precedence (Wu and Filutowicz 1999). Nevertheless, we conclude that, in the presence of TCT, PGRPLC2 and PGRPLC3 show specific coprecipitation with one another.

\section{Discussion}

We demonstrate here that the mosquito midgut is heterogeneous in its expression of immune effectors and at least one immune receptor. In particular, we observe higher expression of the AMPs GAM1, CEC1 and LYSC1 in the cardia region compared with the posterior region of sugar-fed and blood-fed 
midguts. The Drosophila midgut is already understood to be compartmentalized in its immune and digestive function (Buchon and Osman 2015), with enrichment of Imd dependent immune genes in the cardia region (Buchon et al. 2013; Tzou et al. 2000). This likely both ensures that exogenous bacteria entering the gut encounter a robust initial immune response and allows the microbiota in the posterior region to persist. A question arising from this finding is the mechanism by which the gut epithelium mediates this heterogeneity, given that it is exposed to, and indeed responds to, bacterial ligand along its length. Despite observing a very similar spatial heterogeneity in the expression of PGRPLC1, we were not able to detect any clear isoform-specific functionality in this region, perhaps suggesting that PGRPLC receptor variation does not underlie this basal variation in immunogenicity.

Previous structural modelling predicted that dimerization of all isoform-isoform combinations was feasible, except for PGRPLC3 homodimers (Meister et al. 2009). To our knowledge, the experiments presented here are the first to explore in vitro the functionality of the mosquito PGRPLC isoforms. Our results show that PGRPLC1 and PGRPLC3 co-precipitate with polymeric DAP-type peptidoglycan. In the presence of TCT, PGRPLC2 and PGRPLC3 co-precipitate with one another. These data are consistent with a model whereby PGRPLC2 plays a similar role to Drosophila PGRP-LCa, which acts as an adaptor to PGRP-LCx in the presence of TCT, while PGRPLC3 is a PGRP-LCx equivalent.

This model is supported by previously published data on the functionality of the PGRPLC isoforms. All isoforms contribute to resistance to systemic Gram-negative infection, whilst only PGRPLC1 and PGRPLC3 are necessary for resistance to Gram-positive infection (Meister et al. 2009). Given that only Gram-negative bacteria shed TCT, this is consistent with PGRPLC2 only playing a role in the presence of Gram-negatives. In A. gambiae cultured cells, overexpression of either PGRPLC1 or PGRPLC3, but not PGRPLC2, is sufficient to induce CEC1 expression in the absence of infection (Lin et al. 2007). Again, this is consistent with PGRPLC2 acting as an adapter and alone being unable to dimerize with itself.

In the knockdown experiments presented here, we find both PGRPLC2 and PGRPLC3 acting as positive regulators of REL2 responsive AMPs. We also observed GAM1 to be responsive to REL2 after blood feeding, but did not observe knockdown of any single PGRPLC isoform to reproduce this effect. This could suggest that there is redundancy amongst the three PGRPLC isoforms under these conditions, or indeed that PGRPLC is able to dimerize with other partners to stimulate the pathway, such as PGRPLA (Gendrin et al. 2017). This model is also consistent with our observation that PGRPLC3 is not necessary for AMP induction in the sugar-fed gut, suggesting that PGRPLC2 can dimerize with another partner under these conditions.

Interestingly, PGRPLC1 silencing was found to lead to stimulation of the Imd pathway in the anterior part of the midgut. This is intriguing, considering that the same isoform is known to be able to induce the Imd pathway (Lin et al. 2007). However, it has been shown in the Drosophila gut that a single peptidoglycan receptor, PGRP-LE, induces the Imd pathway in response to infectious bacteria, while promoting tolerance to the microbiota via induction of Imd negative regulators (Bosco-Drayon et al. 2012). Drosophila PGRP-LC also has a dual role in Imd induction and negative regulation, depending on its intracellular domain (Neyen et al. 2016).

Together, our data indicate that Anopheles PGRPLC isoforms can discriminate polymeric and monomeric peptidoglycan. They also suggest that PGRPLC2 acts as an adapter to PGRPLC3 for the specific sensing of TCT, leading to the induction of the Imd pathway. Finally, they add a putative regulatory role for PGRPLC1, besides its shown Imd-inducing activity.

\section{Acknowledgements}

We thank Lara Selles-Vidal and Claudia Wyer for technical assistance. FHR was supported by a Biotechnology and Biological Sciences Research Council (BBSRC) doctoral training studentship. The work was funded by the BBSRC Project BB/K009338/1 and the Wellcome Trust Investigator Award 107983/Z/15/Z. 
362 Figure 1. Spatial heterogeneity of AMP and PGRPLC1 expression in the mosquito gut.

363

364

365

366

367

368

369

370

371

372

373

374

375

376

377

378

379

380

381

382

383

384

385

386

387

388

389

390

391

392

393

394

395

396

397

398

399

400

401

402

403

404

405

(A) Schematic of the mosquito gut, with dissected midgut regions indicated. (B) 16S rRNA distribution throughout the gut of sugar-fed and blood-fed females, determined by qRT-PCR on cDNA using universal 16S primers. Each point represents a pool of tissues from 15-30 individual mosquitoes, derived from 5 (blood-fed) and up to 8 (sugar-fed) independent batches of mosquito. Bacterial load significantly increases in the posterior region after blood feeding: $p<0.05$, ANOVA following linear mixed effect regression model fitting. (C-E) Expression of CEC1, GAM1 and LYSC1 throughout the gut of sugar-fed and blood-fed females, with or without antibiotic feeding. Each point represents a pool of tissues from 15-30 individual mosquitoes, derived from 5 (blood-fed) and up to 6 (sugar-fed) independent batches of mosquito. For all three AMPs, tissue of origin had a statistically significant effect on AMP expression level: CEC1 $p<0.001, G A M 1 p<0.001$, LYSC1 $p<0.01$, ANOVA following linear mixed effect regression model fitting. (F) Expression of $P G R P L C 1$ throughout the gut of sugarfed mosquitoes. Each point represents a pool of tissues, of 15-30 individual mosquitoes per pool, derived from 5 independent batches of mosquito. Tissue of origin had a statistically significant effect on PGRPLC1 expression level: $p<0.01$, ANOVA following linear regression model fitting. In graphs B$F$, the shapes of the points for the sugar-fed posterior region indicate whether samples were from the whole posterior region (circles), the proximal half (squares) or the distal half (triangles).

Figure 2. The effect of REL2 and PGRPLC isoform knockdown on GAM1 and LYSC1 expression in the mosquito gut.

(A) GAM1 expression in different regions of the sugar-fed midgut following injection of dsRNA against LACZ (control), REL2 or specific PGRPLC isoforms. REL2 dsRNA causes a significant reduction in GAM1 expression independently of tissue of origin $(p<0.05)$, PGRPLC1 dsRNA causes a significant increase in GAM1 expression in the anterior region $(p<0.05)$, and PGRPLC2 dsRNA causes a significant reduction in GAM1 expression in the cardia $(p<0.05)$ and anterior regions $(p<0.05)$. The shape of the data points indicate whether the sample represents the whole posterior region (circles), the proximal half (squares) or the distal half (triangles). (B) LYSC1 expression in different regions of the blood-fed midgut following injection of dsRNA against LACZ (control), REL2 or specific PGRPLC isoforms. REL2 dsRNA causes a significant reduction in LYSC1 expression in the cardia $(p=0.05)$ and anterior $(p<0.05)$ regions, PGRPLC2 dsRNA causes a significant reduction in LYSC1 expression specifically in the posterior region $(p<0.05)$, and PGRPLC3 dsRNA causes a significant reduction in LYSC1 expression in the cardia $(p<0.05)$ and posterior $(p<0.01)$ regions. In both panels, each point represents a pool of tissues from 10-20 individual mosquitoes, derived from 3 (REL2, PGRPLC1 and PGRPLC2) or 4 (PGRPLC3 and LACZ) independent batches of mosquito. P values are derived from ANOVA tests following linear mixed effect regression model fitting.

\section{Figure 3. PGRPLC1 and PGRPLC3 co-precipitate with polymeric DAP-type peptidoglycan.}

(A) Pull down assays using insoluble, polymeric DAP-type peptidoglycan and single PGRPLC isoforms. Blot shows one of three independent replicates, each giving the same result. (B) Pull down assays using insoluble, polymeric DAP-type peptidoglycan and combinations of PGRPLC isoforms, as indicated. Blot shows one of two independent replicates, both giving the same result. Western blots are non-reducing and were probed with anti-strep tag II antibody (1:1000 in 3\% BSA). In=input (recombinant PGRP that had not been exposed to peptidoglycan); $\mathrm{F}=$ free; B=bound.

\section{Figure 4. PGRPLC2 and PGRPLC3 co-precipitate in the presence of TCT.}

(A-B) The PGRPLC2 ectodomain interacts with the PGRPLC3 ectodomain in the presence of TCT. His-tagged PGRPLC isoforms were incubated with non-His tagged PGRPLC isoforms, pulled down by 
His tag affinity and the free $(F)$ and bound $(B)$ fractions analyzed. Western blots are non-reducing and were probed with anti-strep- tag II antibody (1:1000 in 3\% BSA).

\section{Figure S1. Expression of PGRPLC2 and PGRPLC3 does not vary along the length of the gut.}

(A) Schematic indicating qRT-PCR primer positioning on the PGRPLC transcript. (B) Expression of the PGRPLC2 and PGRPLC3 transcripts throughout the gut of sugar-fed mosquitoes. Each point represents a pool of tissues, of 15-30 individual mosquitoes per pool, derived from 5 independent batches of mosquito.

\section{Figure S2. Knockdown efficiencies of REL2 and PGRPLC isoforms in different regions of the sugar-fed and blood-fed gut.}

Each point represents a pool of tissues, of 10-20 individual mosquitoes per pool, derived from 3 (REL2, PGRPLC1 and PGRPLC2) or 4 (PGRPLC3 and LACZ) independent batches of mosquito. For the sugar-fed posterior region, point shapes indicate whether the sample represents the whole posterior region (circles), the proximal half (squares) or the distal half (triangles). Knockdown was quantified concurrently with AMP expression analysis, $24 \mathrm{~h}$ (sugar-fed) or $48 \mathrm{~h}$ (blood-fed) after dsRNA injection.

\section{Figure S3. The effect of REL2 and PGRPLC isoform knockdown on GAM1, CEC1 and LYSC1 expression in the mosquito gut.}

(A) CEC1 expression in different regions of the sugar-fed midgut following injection of dsRNA against LACZ (control), REL2 or specific PGRPLC isoforms. PGRPLC2 dsRNA causes a significant reduction in CEC1 expression in the cardia region $(p<0.05)$. (B) LYSC1 expression in different regions of the sugar-fed midgut following injection of dsRNA against LACZ (control), REL2 or specific PGRPLC isoforms. (C) GAM1 expression in different regions of the blood-fed midgut following injection of dsRNA against LACZ (control), REL2 or specific PGRPLC isoforms. REL2 dsRNA causes a significant reduction in GAM1 expression independently of tissue of origin $(p<0.05)$. (D) CEC1 expression in different regions of the blood-fed midgut following injection of dsRNA against LACZ (control), REL2 or specific PGRPLC isoforms. (A-D) Each point represents a pool of tissues, of 10-20 individual mosquitoes per pool, derived from 3 (REL2, PGRPLC1 and PGRPLC2) or 4 (PGRPLC3 and LACZ) independent batches of mosquito. $p$ values are the result of ANOVAs following linear mixed effect regression model fitting. For the sugar-fed posterior region, point shapes indicate whether the sample represents the whole posterior region (circles), the proximal half (squares) or the distal half (triangles).

\section{Figure S4. Production of recombinant PGRPLC ectodomains.}

(A) Schematic of the expression region in the pIEX-10 vector used for recombinant protein production. (B) Coomassie stained gel of purified proteins before and after His-tag cleavage. (C) Glycosylation sites predicted using the GlycoEP server (Chauhan, Rao, and Raghava 2013) using Binary Profile of Patterns (BPP) prediction with SVM threshold set to 0.0. (D) PNGase F treatment of recombinant PGRPs. Western blot probed with anti His antibody (1:2000 in 3\% BSA).

\section{References}

Aggarwal, K., F. Rus, C. Vriesema-Magnuson, D. Erturk-Hasdemir, N. Paquette, and N. Silverman. 2008. 'Rudra interrupts receptor signaling complexes to negatively regulate the IMD pathway', PLoS Pathog, 4: e1000120.

Basbous, N., F. Coste, P. Leone, R. Vincentelli, J. Royet, C. Kellenberger, and A. Roussel. 2011. 'The Drosophila peptidoglycan-recognition protein LF interacts with peptidoglycan-recognition protein LC to downregulate the Imd pathway', EMBO Rep, 12: 327-33. 
Bosco-Drayon, V., M. Poidevin, I. G. Boneca, K. Narbonne-Reveau, J. Royet, and B. Charroux. 2012. 'Peptidoglycan sensing by the receptor PGRP-LE in the Drosophila gut induces immune responses to infectious bacteria and tolerance to microbiota', Cell Host Microbe, 12: 153-65.

Buchon, N., and D. Osman. 2015. 'All for one and one for all: Regionalization of the Drosophila intestine', Insect Biochem Mol Biol, 67: 2-8.

Buchon, N., D. Osman, F. P. David, H. Y. Fang, J. P. Boquete, B. Deplancke, and B. Lemaitre. 2013. 'Morphological and molecular characterization of adult midgut compartmentalization in Drosophila', Cell Rep, 3: 1725-38.

Chang, C. I., Y. Chelliah, D. Borek, D. Mengin-Lecreulx, and J. Deisenhofer. 2006. 'Structure of tracheal cytotoxin in complex with a heterodimeric pattern-recognition receptor', Science, 311: 1761-4.

Chang, C. I., K. Ihara, Y. Chelliah, D. Mengin-Lecreulx, S. Wakatsuki, and J. Deisenhofer. 2005. 'Structure of the ectodomain of Drosophila peptidoglycan-recognition protein LCa suggests a molecular mechanism for pattern recognition', Proc Natl Acad Sci U S A, 102: 10279-84.

Chauhan, J. S., A. Rao, and G. P. Raghava. 2013. 'In silico platform for prediction of N-, O- and Cglycosites in eukaryotic protein sequences', PLoS One, 8: e67008.

Christophides, G. K., E. Zdobnov, C. Barillas-Mury, E. Birney, S. Blandin, C. Blass, P. T. Brey, F. H. Collins, A. Danielli, G. Dimopoulos, C. Hetru, N. T. Hoa, J. A. Hoffmann, S. M. Kanzok, I. Letunic, E. A. Levashina, T. G. Loukeris, G. Lycett, S. Meister, K. Michel, L. F. Moita, H. M. Muller, M. A. Osta, S. M. Paskewitz, J. M. Reichhart, A. Rzhetsky, L. Troxler, K. D. Vernick, D. Vlachou, J. Volz, C. von Mering, J. Xu, L. Zheng, P. Bork, and F. C. Kafatos. 2002. 'Immunity-related genes and gene families in Anopheles gambiae', Science, 298: 159-65.

Costechareyre, D., F. Capo, A. Fabre, D. Chaduli, C. Kellenberger, A. Roussel, B. Charroux, and J. Royet. 2016. 'Tissue-Specific Regulation of Drosophila NF-x03BA;B Pathway Activation by Peptidoglycan Recognition Protein SC', J Innate Immun, 8: 67-80.

Dong, Y., F. Manfredini, and G. Dimopoulos. 2009. 'Implication of the mosquito midgut microbiota in the defense against malaria parasites', PLoS Pathog, 5: e1000423.

Gendrin, M., F. Turlure, F. H. Rodgers, A. Cohuet, I. Morlais, and G. K. Christophides. 2017. 'The Peptidoglycan Recognition Proteins PGRPLA and PGRPLB Regulate Anopheles Immunity to Bacteria and Affect Infection by Plasmodium', J Innate Immun, 9: 333-42.

Kamareddine, L., W. P. Robins, C. D. Berkey, J. J. Mekalanos, and P. I. Watnick. 2018. 'The Drosophila Immune Deficiency Pathway Modulates Enteroendocrine Function and Host Metabolism', Cell Metab, 28: 449-62.e5.

Kaneko, T., W. E. Goldman, P. Mellroth, H. Steiner, K. Fukase, S. Kusumoto, W. Harley, A. Fox, D. Golenbock, and N. Silverman. 2004. 'Monomeric and polymeric gram-negative peptidoglycan but not purified LPS stimulate the Drosophila IMD pathway', Immunity, 20: 637-49.

Kaneko, T., T. Yano, K. Aggarwal, J. H. Lim, K. Ueda, Y. Oshima, C. Peach, D. Erturk-Hasdemir, W. E. Goldman, B. H. Oh, S. Kurata, and N. Silverman. 2006. 'PGRP-LC and PGRP-LE have essential yet distinct functions in the drosophila immune response to monomeric DAP-type peptidoglycan', Nat Immunol, 7: 715-23.

Kleino, A., H. Myllymaki, J. Kallio, L. M. Vanha-aho, K. Oksanen, J. Ulvila, D. Hultmark, S. Valanne, and M. Ramet. 2008. 'Pirk is a negative regulator of the Drosophila Imd pathway', J Immunol, 180: 5413-22.

Leulier, F., C. Parquet, S. Pili-Floury, J. H. Ryu, M. Caroff, W. J. Lee, D. Mengin-Lecreulx, and B. Lemaitre. 2003. 'The Drosophila immune system detects bacteria through specific peptidoglycan recognition', Nat Immunol, 4: 478-84.

Lim, J. H., M. S. Kim, H. E. Kim, T. Yano, Y. Oshima, K. Aggarwal, W. E. Goldman, N. Silverman, S. Kurata, and B. H. Oh. 2006. 'Structural basis for preferential recognition of diaminopimelic acid-type peptidoglycan by a subset of peptidoglycan recognition proteins', J Biol Chem, 281: 8286-95.

Lin, H. U. I., Lingmin Zhang, Coralia Luna, Ngo T. Hoa, and Liangbiao Zheng. 2007. 'A splice variant of PGRP-LC required for expression of antimicrobial peptides in Anopheles gambiae', Insect Science, 14: 185-92. 
501

502

503

504

505

506

507

508

509

510

511

512

513

514

515

516

517

518

519

520

521

522

523

524

525

526

527

528

529

530

531

532

533

534

535

536

537

Maillet, F., V. Bischoff, C. Vignal, J. Hoffmann, and J. Royet. 2008. 'The Drosophila peptidoglycan recognition protein PGRP-LF blocks PGRP-LC and IMD/JNK pathway activation', Cell Host Microbe, 3: 293-303.

Meister, S., B. Agianian, F. Turlure, A. Relogio, I. Morlais, F. C. Kafatos, and G. K. Christophides. 2009. 'Anopheles gambiae PGRPLC-mediated defense against bacteria modulates infections with malaria parasites', PLoS Pathog, 5: e1000542.

Mellroth, P., J. Karlsson, J. Hakansson, N. Schultz, W. E. Goldman, and H. Steiner. 2005. 'Ligandinduced dimerization of Drosophila peptidoglycan recognition proteins in vitro', Proc Natl Acad Sci U S A, 102: 6455-60.

Neyen, C., M. Poidevin, A. Roussel, and B. Lemaitre. 2012. 'Tissue- and ligand-specific sensing of gram-negative infection in drosophila by PGRP-LC isoforms and PGRP-LE', J Immunol, 189: 188697.

Neyen, C., C. Runchel, F. Schupfer, P. Meier, and B. Lemaitre. 2016. 'The regulatory isoform rPGRPLC induces immune resolution via endosomal degradation of receptors', Nat Immunol, 17: 1150-8.

Paredes, J. C., D. P. Welchman, M. Poidevin, and B. Lemaitre. 2011. 'Negative regulation by amidase PGRPs shapes the Drosophila antibacterial response and protects the fly from innocuous infection', Immunity, 35: 770-9.

Persson, C., S. Oldenvi, and H. Steiner. 2007. 'Peptidoglycan recognition protein LF: a negative regulator of Drosophila immunity', Insect Biochem Mol Biol, 37: 1309-16.

Ryu, J. H., S. H. Kim, H. Y. Lee, J. Y. Bai, Y. D. Nam, J. W. Bae, D. G. Lee, S. C. Shin, E. M. Ha, and W. J. Lee. 2008. 'Innate immune homeostasis by the homeobox gene caudal and commensal-gut mutualism in Drosophila', Science, 319: 777-82.

Stenbak, C. R., J. H. Ryu, F. Leulier, S. Pili-Floury, C. Parquet, M. Hervé, C. Chaput, I. G. Boneca, W. J. Lee, B. Lemaitre, and D. Mengin-Lecreulx. 2004. 'Peptidoglycan molecular requirements allowing detection by the Drosophila immune deficiency pathway', J Immunol, 173: 7339-48.

Tzou, P., S. Ohresser, D. Ferrandon, M. Capovilla, J. M. Reichhart, B. Lemaitre, J. A. Hoffmann, and J. L. Imler. 2000. 'Tissue-specific inducible expression of antimicrobial peptide genes in Drosophila surface epithelia', Immunity, 13: 737-48.

Werner, T., K. Borge-Renberg, P. Mellroth, H. Steiner, and D. Hultmark. 2003. 'Functional diversity of the Drosophila PGRP-LC gene cluster in the response to lipopolysaccharide and peptidoglycan', J Biol Chem, 278: 26319-22.

Wu, J., and M. Filutowicz. 1999. 'Hexahistidine (His6)-tag dependent protein dimerization: a cautionary tale', Acta Biochim Pol, 46: 591-9.

Zaidman-Remy, A., M. Herve, M. Poidevin, S. Pili-Floury, M. S. Kim, D. Blanot, B. H. Oh, R. Ueda, D. Mengin-Lecreulx, and B. Lemaitre. 2006. 'The Drosophila amidase PGRP-LB modulates the immune response to bacterial infection', Immunity, 24: 463-73. 
A

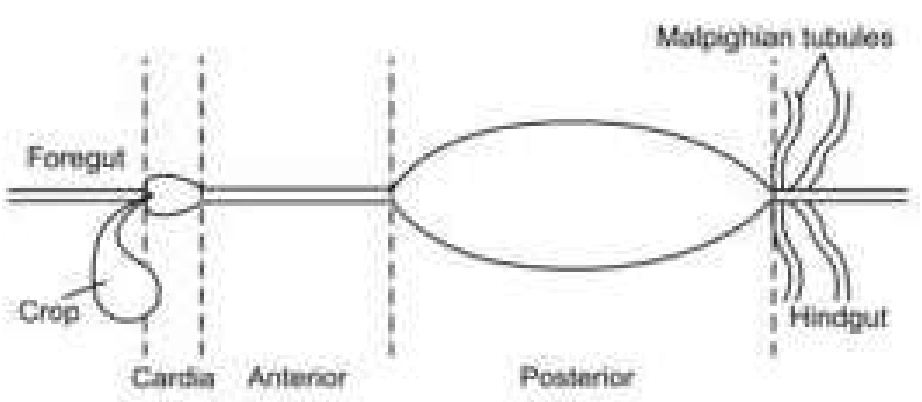

C

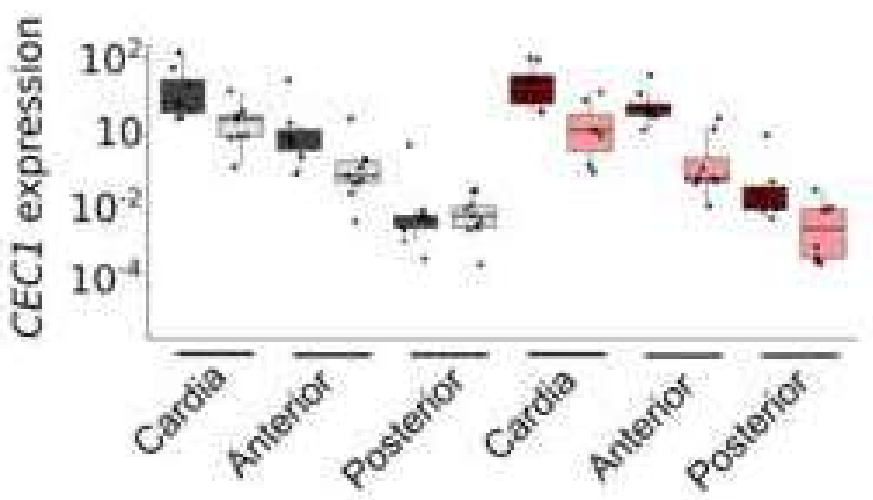

Sugar-fed Blood-fed

E

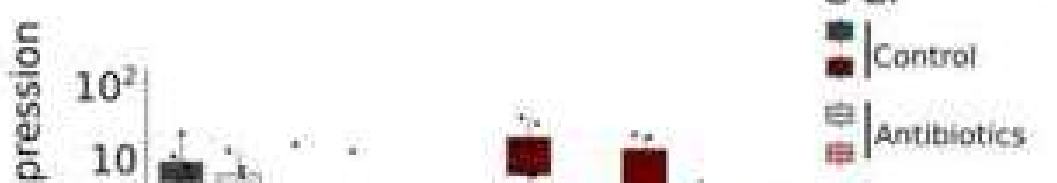

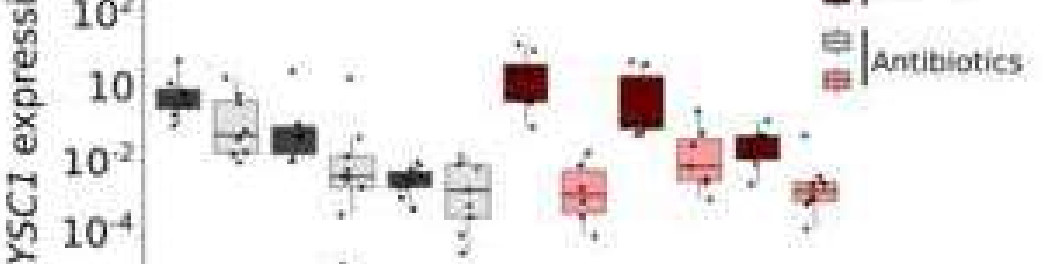

पू

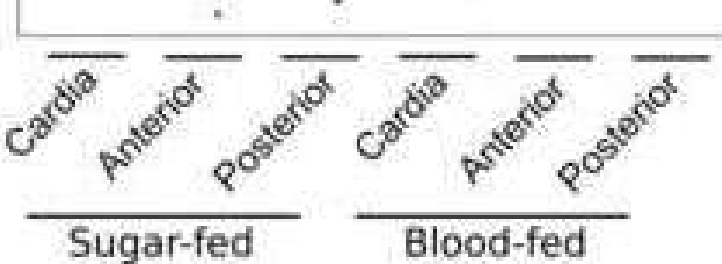

B

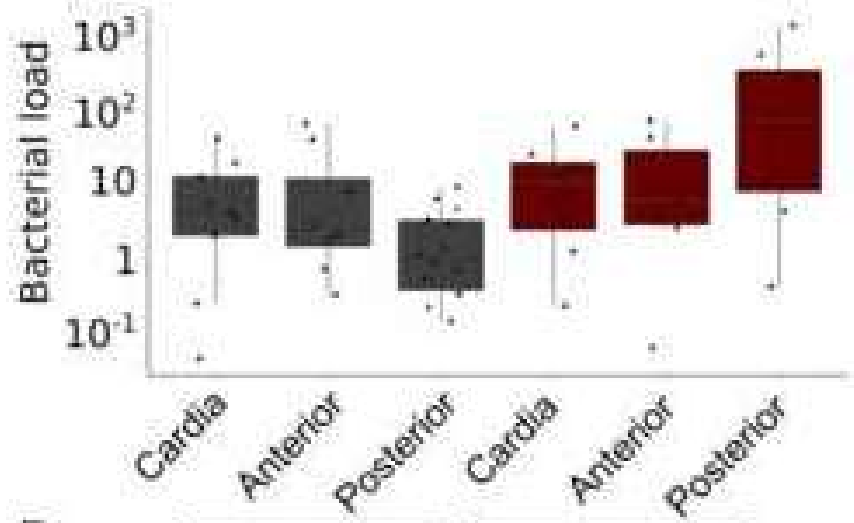

D

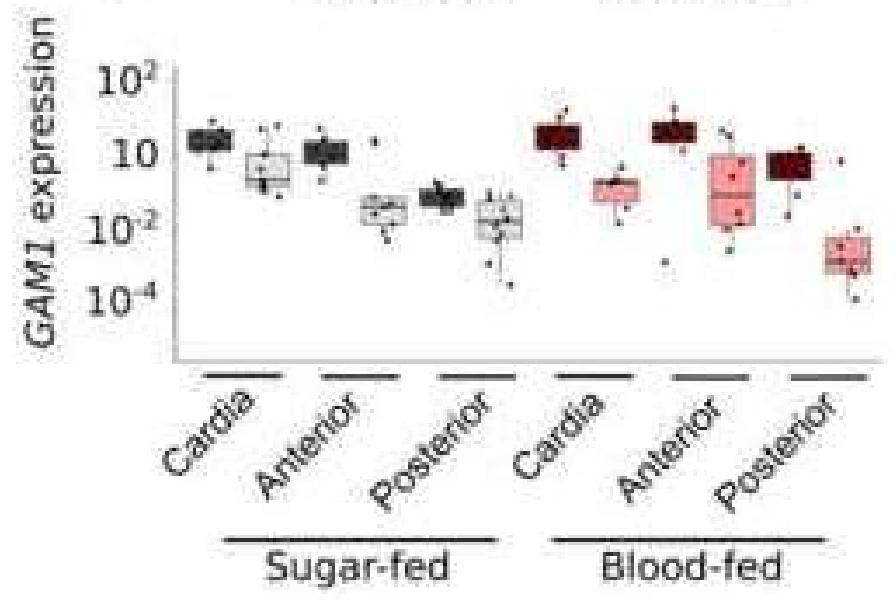

$\mathrm{F}$

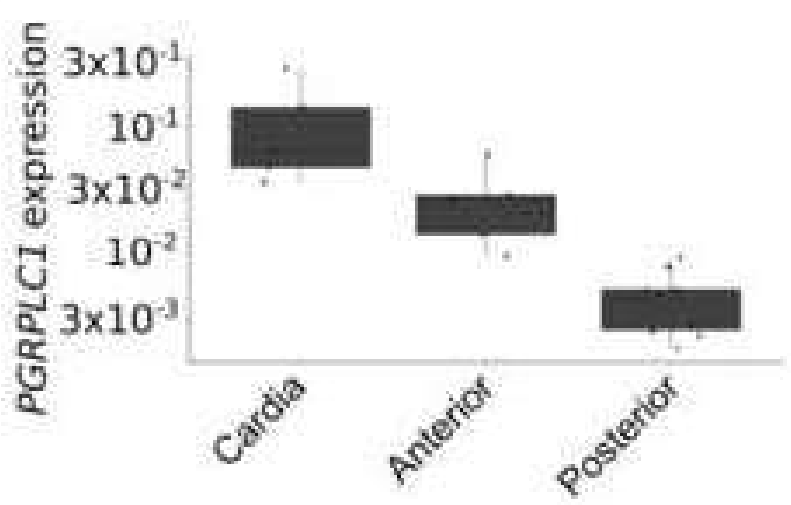




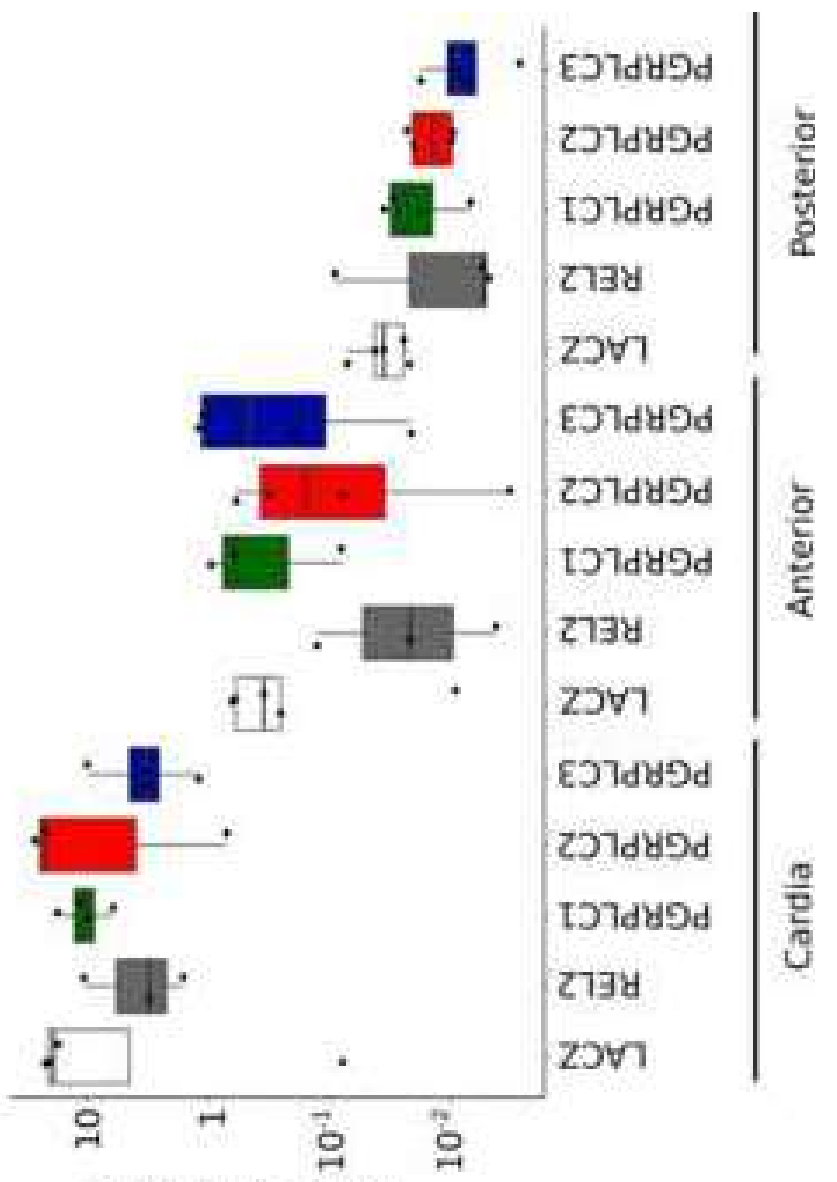

$\infty$ voissaddxa [0SA7

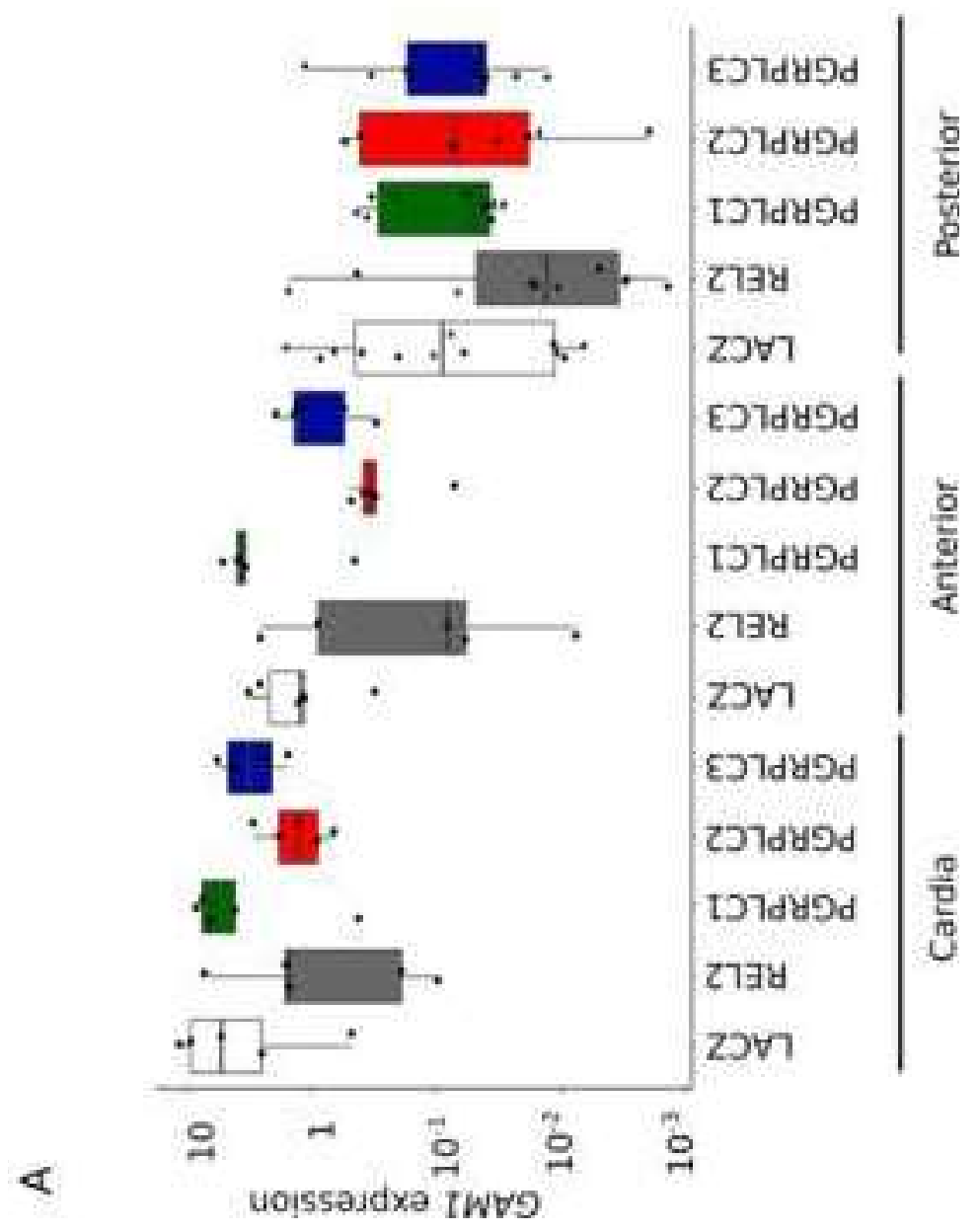




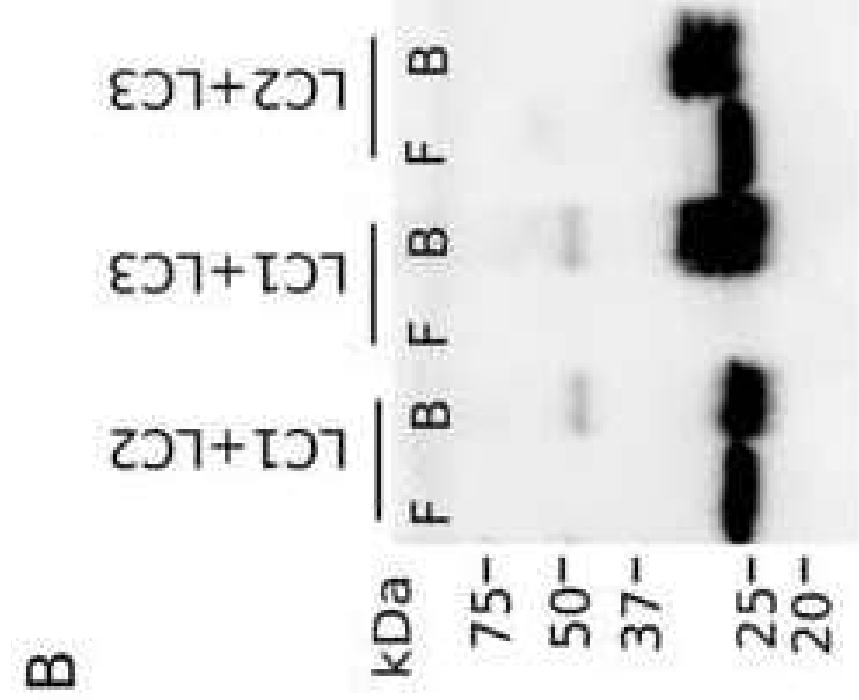

|

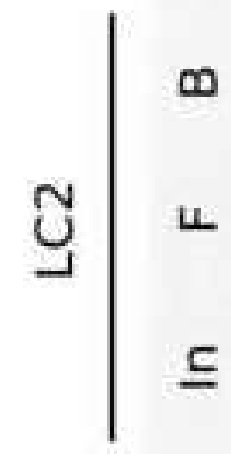

$\left.\right|^{\infty}$

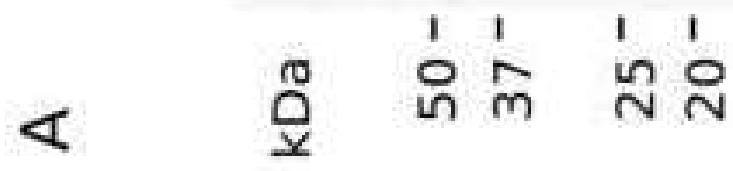




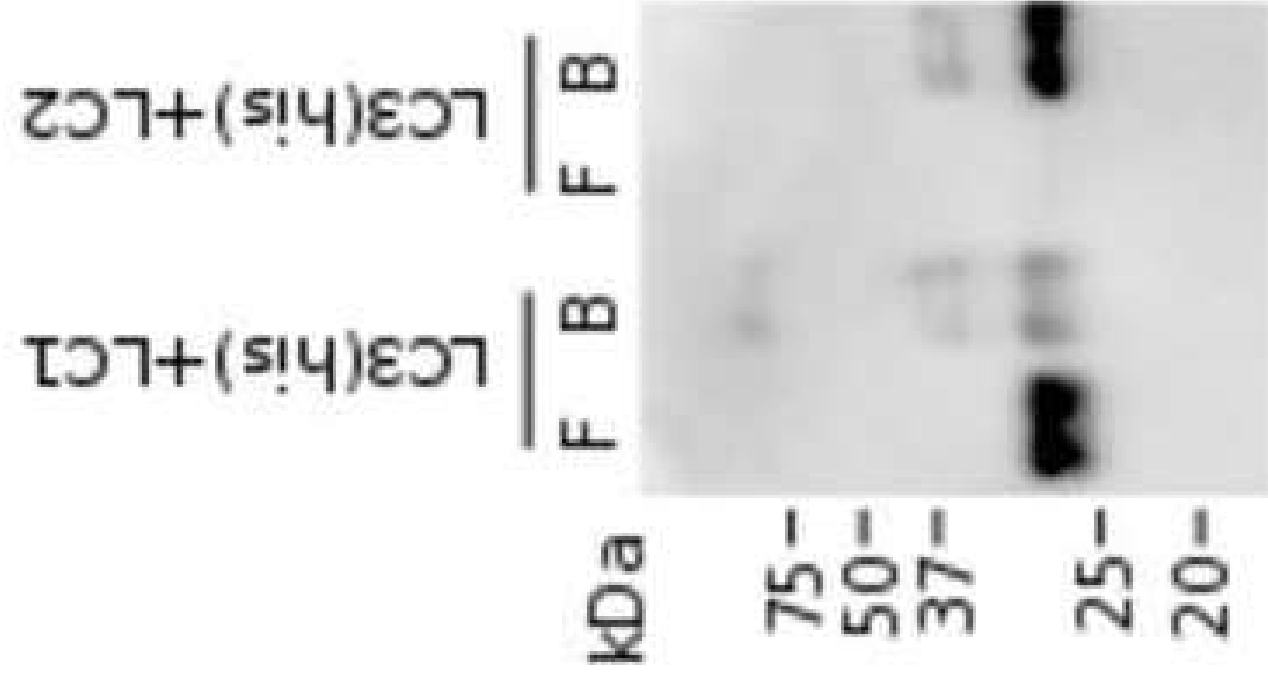

$\infty$

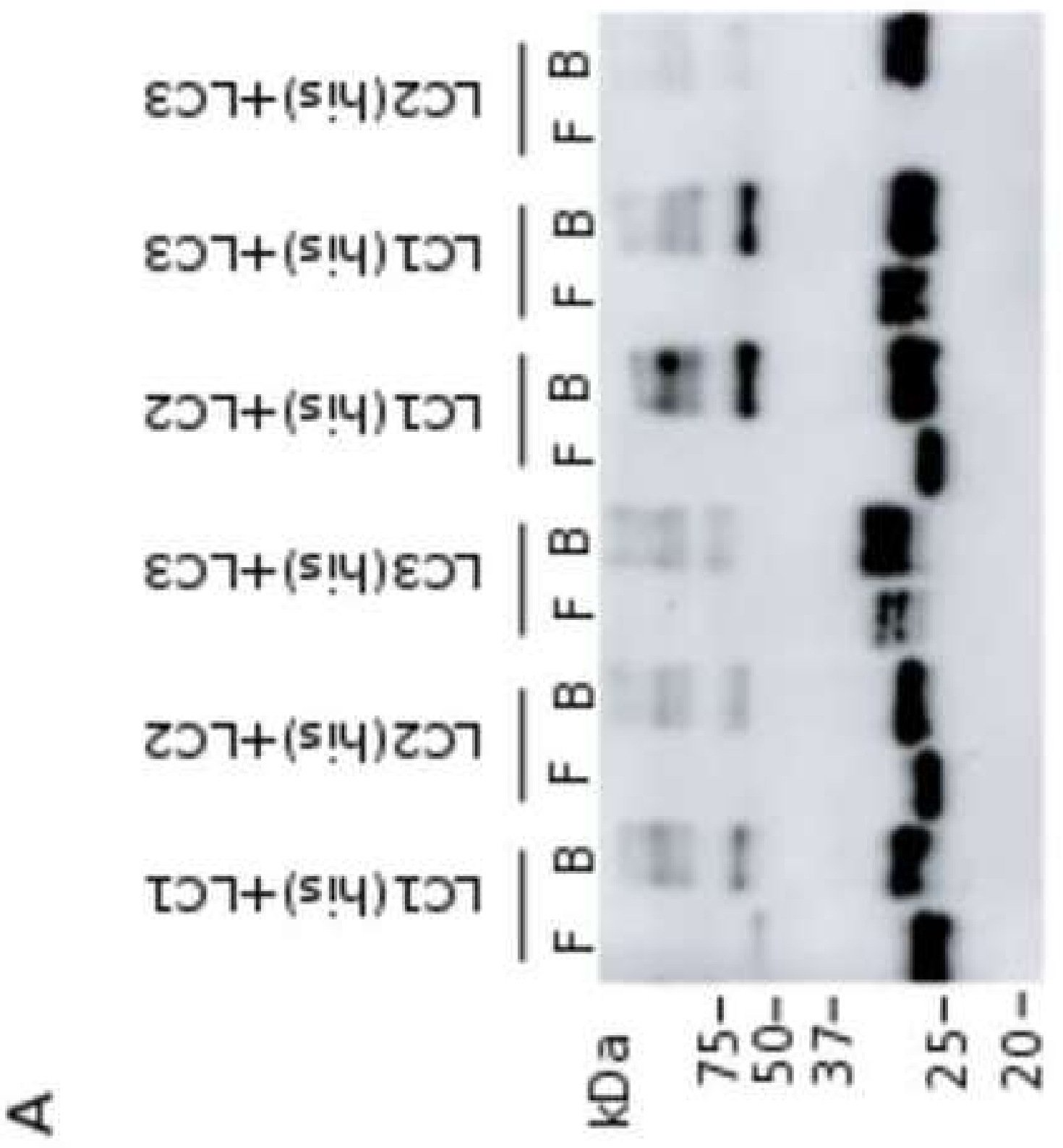

